\title{
Management of brain abscesses associated with halo fixation
}

\author{
Case report
}

\author{
Toba Niazi, M.D., ${ }^{1}$ Alfredo Quinoñes-Hinojosa, M.D., ${ }^{3}$ Meic H. Schmidt, M.D. ${ }^{1,2}$ \\ ${ }^{1}$ Department of Neurosurgery and ${ }^{2}$ Huntsman Cancer Institute, University of Utah, Salt Lake City, \\ Utah; and ${ }^{3}$ Department of Neurological Surgery, Johns Hopkins University, Baltimore, Maryland
}

\begin{abstract}
$\checkmark$ Halo orthoses are commonly used in the management of a variety of cervical spinal pathological conditions. Although placement of the cranial pins was initially believed to be safe with minimal complications, minor complications related to the cranial pins have the potential to create a setting for formation of cerebral abscesses. The risk of death due to cerebral abscesses has declined in the modern antibiotic era, but cerebral abscesses are associated with long-standing neurological morbidity that should not be considered negligible. Prevention by careful pin placement and hygiene and appropriate early management are crucial to prevent more serious complications. The authors report the case of a patient treated with a halo orthosis after incurring cervical spinal trauma who developed a cerebral abscess as a complication related to the cranial pins of the halo fixation device. They review the literature in an attempt to formulate a standardized treatment algorithm to prevent this disease process and to treat an abscess if it should form.
\end{abstract} (DOI: 10.3171/FOC/2008/24/6/E14)

\section{KEY WORDS - cerebral abscess - cranial pin - halo orthosis - inner table of skull}

$\mathrm{H}$ ALO orthoses are used routinely in both neurosurgical and orthopedic specialties for management of a variety of cervical spinal pathological conditions, including but not limited to cervical instability secondary to trauma, degenerative disease states, neoplasms, and scoliosis, as well as after surgical reconstruction to maximize the fusion process. After pioneering the initial halo device in 1959, Perry and Nicke ${ }^{10}$ recognized the drawbacks associated with the pins used in affixing the halo device to the skull and detailed a regimen to prevent complications of halo fixation. They emphasized that careful and appropriate initial placement of pins and subsequent meticulous hygiene of the pin sites was imperative to prevent any problems with the fixation device. Despite initial reports $1,2,4,5$ that associated complications were relatively infrequent and were usually minor problems relating specifically to the pin sites, subsequent reports ${ }^{1-6,11,12}$ made it clear that catastrophic outcomes were possible. Cerebral brain abscesses in association with the halo fixation device have been reported in the literature in 17 cases..$^{15}$ Although the risk of death due to cerebral abscesses has declined since the introduction of antibiotics, this disease process is associated with long-standing neurological morbidity that should not be considered negligible. ${ }^{12,15}$ We illustrate a case of a cerebral abscess that developed as a direct result of pin site-related complications and review the literature in an attempt to create a standardized treatment algorithm to prevent this process or to treat it if it should occur.

\section{Case Report}

History and Presentation. This 40-year-old woman with a history of alcoholism and cocaine dependency presented to the University of Utah emergency room after a motor vehicle collision complaining of cervical spine tenderness and having experienced transient left-arm paresthesias immediately after the accident.

Examination. On examination, the patient was alert, awake, oriented, and hemodynamically stable, with no deficits in motor or sensory function. The results of a trauma work up were unremarkable with the exception of findings on the CT and CT angiograms of her cervical spine. These studies demonstrated a comminuted fracture through the second vertebral body and displacement of the body anteriorly by 2.5 millimeters with associated fracture lines involving bilateral transverse foramina and no evidence of vertebral artery injury or impedance of flow (Fig. 1A-C). There was no significant central canal impingement or narrowing.

Initial Treatment and Posttreatment Course. The patient was fitted with a halo device with no change in her motor or sensory function; she remained clinically stable and was discharged the next day after appropriate halo care was described.

The patient missed her follow-up appointment but presented 7 weeks later with complaints of headaches and pustular exudate from the right frontal pin site and a loose pin at this site. This pin was removed and the area around it was thoroughly cleansed.

The patient underwent a CT scan of her head with and without administration of a contrast agent to ensure that there was no underlying disease process. The CT scan showed no evidence of an osseous or destructive lesion of 

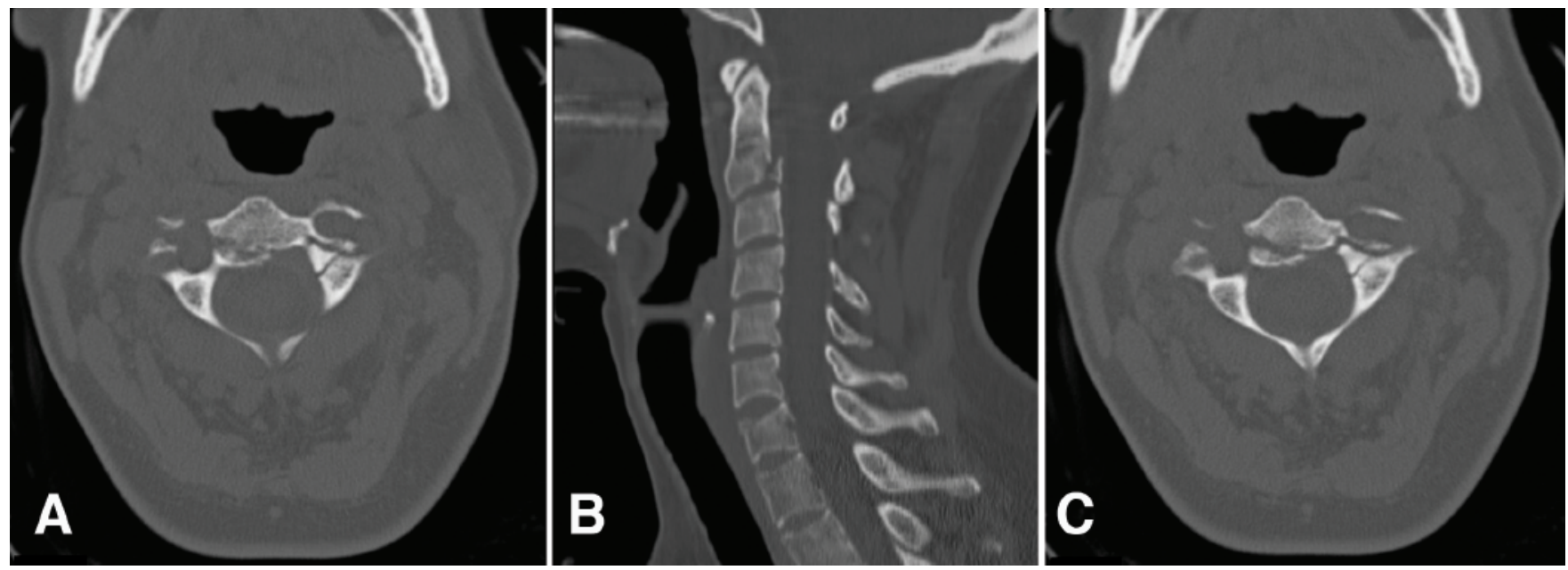

FIG. 1. Axial CT scans (A and C) of the cervical spine and sagittal reconstruction (B) demonstrating a comminuted fracture through the second vertebra and displacement of the body anteriorly by $2.5 \mathrm{~mm}$ with associated fracture lines involving bilateral transverse foramina and no evidence of vertebral artery injury or impedance of flow.

the right frontal bone and no evidence of soft tissue changes to suggest an underlying infection. The patient was treated with a 5-day course of cephalexin to help with the superficial cellulitis and purulent drainage from the previous pin site.

Five weeks later, the patient returned to the clinic with complaints of worsening orbital headaches and continued purulent drainage from both the previous pin site in the right frontal region and the newly placed pin site. The drainage had become more productive since the last visit. A contrast-enhanced CT scan (Fig. 2) of her head demonstrated 2 low-density ring-enhancing lesions in the right frontal lobe. The lesions measured 3.4 and $1.0 \mathrm{~cm}$ in maximum diameter, with associated vasogenic edema and 7 $\mathrm{mm}$ of right-to-left midline shift with subfalcine herniation consistent with cerebral abscess. The right frontal bone in the area of the previous pin and the new pin site demonstrated a permeative lucency indicative of osteomyelitis (Fig. 3).

Operation and Postoperative Course. The patient underwent emergent evacuation of her right frontal abscesses. She was intubated and sedated, and the halo was removed. Fluoroscopy was performed, and lateral neutral and flexion and extension views demonstrated no subluxation and confirmed fusion of the fracture. A right frontal craniotomy was then performed, and the abscess cavity was identified; frank pus and necrotic debris were sent for Gram staining, culturing, and sensitivity testing. A clear communication between the abscess and skull defect from the pin site was noted intraoperatively. Intraoperative bacterial culture was positive for Streptococcus milleri.

After surgery, the patient was treated with intravenous antibiotic therapy for 6 weeks. Unfortunately, the patient began to experience seizures 2 weeks after the evacuation of her cerebral abscesses. She remains on a regimen of antiepileptic medication but continues to have 1-2 seizures per month despite maximal medical treatment.

\section{Discussion and Review of the Literature}

External halo devices have been used since the late 1950s to obtain cervical spinal stabilization. While providing immobilization with maintenance of reduction to achieve healing from a fracture or reconstruction, halo devices allow early mobilization and a shorter hospital course for the patient. ${ }^{15}$ As with every procedure, however, there are inherent risks involved in the use of halo orthoses.

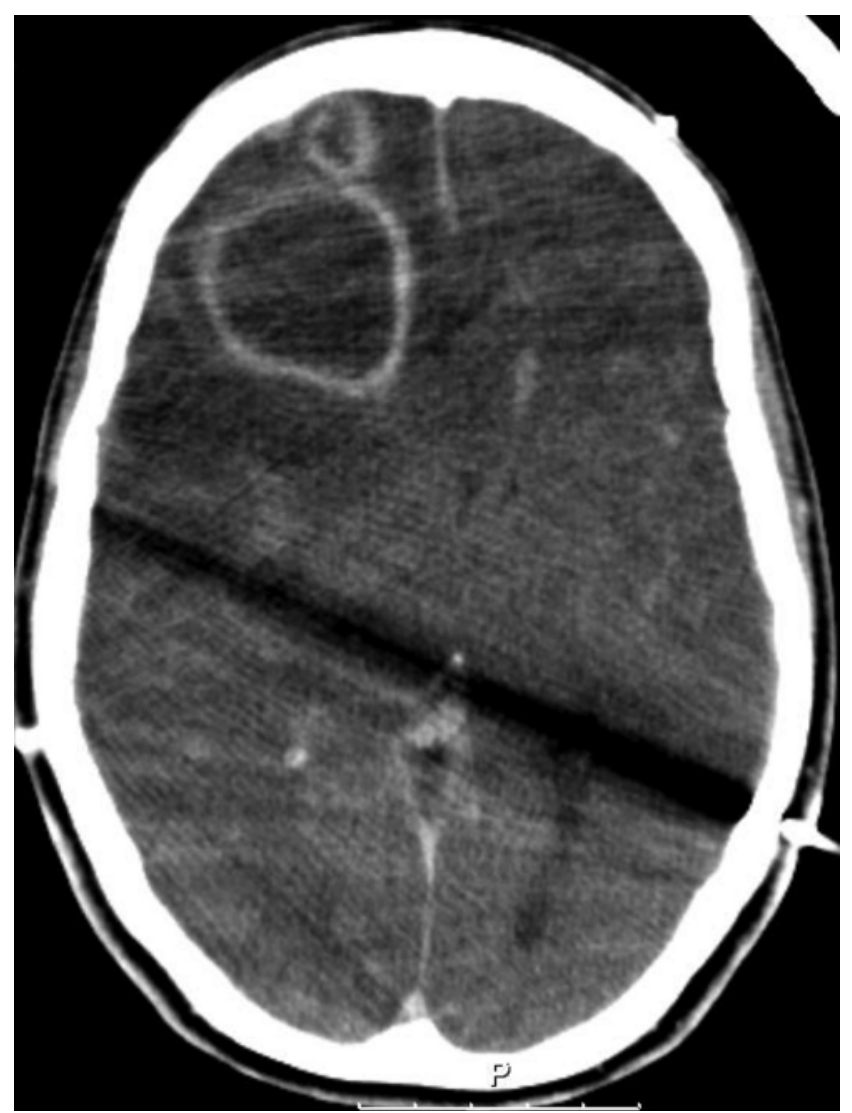

FIG. 2. Contrast-enhanced CT scan of the head demonstrating two low-density ring-enhancing lesions in the right frontal lobe. 
Garfin et al. ${ }^{3}$ reviewed 179 cases to identify the risks associated with the external halo device. The authors found that pin loosening occurred in $36 \%$ of patients, pin site infection in $20 \%$, severe pin discomfort in $18 \%$, dysphagia in $2 \%$, and dural penetration in $1 \%$. Furthermore, although the use of external halo devices is generally thought to be safe, an associated rare complication, cerebral brain abscess, can be fatal. A cerebral abscess is usually incited by the placement, hygiene, loosening, and retightening of the cranial pins. ${ }^{11}$

Appropriate pin site placement is of paramount importance to avoid minor complications that can lead to a cerebral abscess. ${ }^{3,9}$ Pin penetration through the inner table of the skull can create a direct extension into the intracranial cavity and encourage formation of intracranial infections. Pins are engineered so that there is a sharp point and a broader body to prevent the penetration of the inner table of the skull, but penetration still occurs. Garfin et al. ${ }^{3}$ recommended "safe" zones for the placement of pins, avoiding the region of the temporalis muscle. Although the scar created by positioning at the temporalis muscle is within the hairline and thus more aesthetic, penetration of pins through the temporalis muscle is painful and may impede the ability to chew without significant discomfort. The underlying bone in this region is also not suitable for appropriate pin purchase because the squamous part of the temporal bone naturally thins out and is prone to puncture into the intracranial space with repeated pin loosening. Instead, Garfin et al. ${ }^{3}$ recommended placement of the posterior pins at the posterolateral aspects of the calvaria where the density of bone is greater, specifically at an area corresponding to the 4 o'clock and 8 o'clock positions, with 12 o'clock representing the anterior calvaria or glabella and 6 o'clock representing the posterior calvaria or posterior occipital protuberance. Because of the difficulty in achieving firm pin placement, the use of halo traction devices should be avoided in patients who have undergone previous cranial surgery or had recent skull fractures and in those with pathologically soft bone, such as patients with rheumatoid arthritis or multiple myeloma. ${ }^{9}$

Appropriate torque must be applied to the pins during insertion to prevent subsequent loosening and associated infection while avoiding penetration of the inner table of the skull. Rizzolo et al. ${ }^{13}$ performed a prospective randomized trial of 102 patients who were placed in a halo vest with pins inserted at either 6 inch-lbs of torque or 8 inchlbs of torque. Those patients with pins inserted at 6 inch-lbs of torque experienced fewer halo pin complications, including loosening, infection, pin change, and loss of pin purchase. For this reason, the authors recommended that halo pins be inserted with 6 inch-lbs of torque to prevent complications but primarily to prevent penetration of the skull past the diploic space. ${ }^{13}$

Pin site hygiene is crucial because poor hygiene can lead to a local cellulitis, which can track through the skull defect. If there is also a breach of the inner table of the skull, this promotes the formation of an infectious process in the intracranial space. ${ }^{11}$ Water and mild soap are best for cleaning the pin sites. Other cleansing agents traditionally used in routine pin care (povidone-iodine, hydrogen peroxide, chlorhexidine) may have a higher propensity to cause infection based on a sample of patients. ${ }^{8}$ Excessive

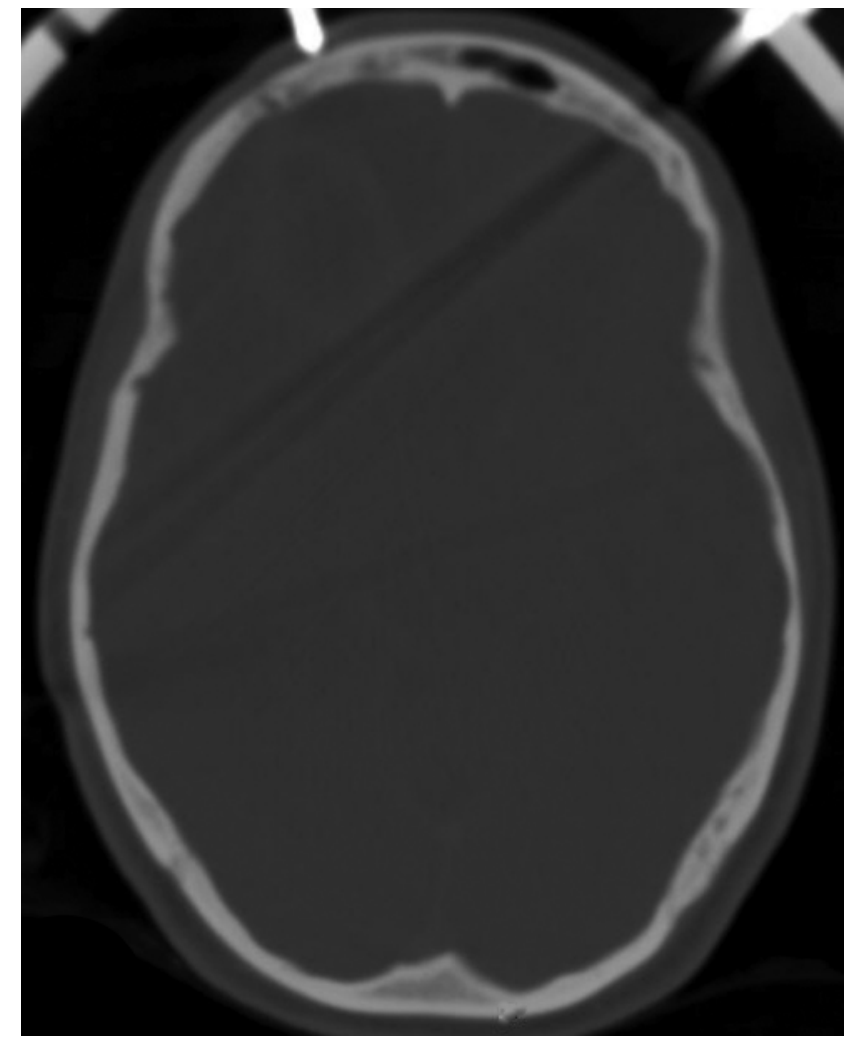

FIG. 3. Axial CT scan showing the right frontal bone in the area of the previous pin and new pin site demonstrating a permeative lucency indicating osteomyelitis.

pin site cleansing can also be problematic and may lead to excess granulation tissue around the pins and subsequent loosening. ${ }^{17}$ It is imperative to provide appropriate instructions regarding pin site hygiene, not only to the patient, but also to the caregiver, who will generally be the primary person involved in pin care and hygiene.

Once the halo device is placed, it is most prudent to retighten the pins 24 hours after placement and then again 1 week later and to provide routine follow-up care at 4- to 6-week intervals. ${ }^{11}$ Delayed and excessive pin tightening should be avoided as either can be associated with compromise of the skull past the diploic space. ${ }^{11}$ If purulent drainage or neurological symptoms are noted, CT of the head should be performed to assess for any penetration through the skull and to look for infection, even if the patient has no specific neurological complaints. If there are no underlying pathological conditions, the existing screw can be removed, the area around it thoroughly cleansed, and a new pin placed at a site remote from the original pin site. If the halo is no longer needed for stabilization, it should be removed altogether. A course of oral antibiotic therapy should also be administered to treat the existing cellulitis at the old pin site and prevent it from spreading.

When a patient with a halo device presents with signs and symptoms suggestive of cerebritis or cerebral abscess, CT of the head, with contrast, should be performed immediately to identify this process in the early stages. In the cases described in the literature, patients with cerebral abscesses have presented with a broad spectrum of symp- 
toms including but not limited to fever, headache, nausea, vomiting, seizure, aphasia, lethargy, meningismus, and psychosis. ${ }^{1-9,11,12,14-17}$ Early recognition is imperative because intravenous antibiotic treatment will be more effective at an earlier stage when only cerebritis is present. ${ }^{17}$ Once the cerebritis progresses to actual abscess formation, a firm fibrous capsule forms containing necrotic material and debris that are resistant to intravenous antibiotic therapy. At this point, surgical evacuation followed by antibiotic therapy is the only option available for treatment. ${ }^{1,17}$ As alluded to above, the cerebral abscess arises from the intracranial extension of cutaneous bacteria present at the pin sites. ${ }^{1,17}$ With penetration through the inner table, the pin provides a point of constant pressure on the surface of the dura mater, and an inflammatory process allows entry of infectious organisms into the subdural space. ${ }^{1}$ Retrograde thrombophlebitis through the dural veins can account for the deeper cerebral abscesses that are seen with halo fixation devices. ${ }^{1}$

Even if they are adequately treated, cerebral abscesses can be associated with serious long-term consequences. Damage to the cerebral parenchyma disturbs the normal propagation of electrical impulses to and from the cerebrum and can serve as a potential seizure focus. This can necessitate long-term antiepileptic treatment as was necessary in our case. Seizures can develop without the presence of an abscess and with pin penetration alone. Nottmeier and Bondurant $^{7}$ described the case of a 29 -year-old man who presented with new-onset tonic-clonic seizures 11 years after the removal of halo orthoses. The previously placed pin had breached the inner table of the skull and created an area of scar tissue that served as the seizure focus 11 years later. Neurological deficits can persist if the infectious process affects an eloquent region of the brain parenchyma. These deficits will lessen over time but will never fully abate.

\section{Conclusions and Recommendations}

Once a halo fixation device is placed, special attention must be focused on the placement, hygiene, and maintenance of the cranial pins to avoid minor complications that can lead to the more devastating complication of a cerebral brain abscess. On the basis of the cases described in the literature and our own institutional experience, we have developed the following recommendations for the use of halo fixation devices and the management of any resultant cerebral abscesses: 1) The halo orthosis should only be used in patients without any underlying skull pathological conditions, including prior craniotomy, skull fracture, or pathologically soft bone. 2) Cranial pins should be seated with 6 inch-lbs of torque and should be placed in the "safe" areas to avoid thin bone that is more prone to penetration into the intracranial space. The pins should be rechecked and retightened at 24 hours and 1 week after placement, with routine follow-up at 4- to 6-week intervals. 3) The importance of proper pin site hygiene, including the potential severity of complications, must be stressed to both the patient and the caregiver. 4) Delayed and excessive pin tightening should be avoided. If purulent drainage or neu- rological symptoms are noted, imaging studies of the head should be performed. If no underlying pathological condition is present, a new cranial pin should be placed at a different locale. A short course of oral antibiotic treatment for the dermal cellulitis is recommended. 5) If an abscess is suspected on the basis of imaging studies, the use of the halo should be discontinued, and cultures of the purulent material should be analyzed. Appropriate antibiotic therapy should be initiated. If the abscess is not amenable to conservative treatment with intravenous antibiotics, surgical evacuation is warranted.

\section{References}

1. Celli P, Palatinsky E: Brain abscess as a complication of cranial traction. Surg Neurol 23:594-596, 1985

2. Dennis GC, Clifton GL: Brain abscess as a complication of halo fixation. Neurosurgery 10:760-761, 1982

3. Garfin SR, Botte MJ, Waters RL, Nickel VL: Complications in the use of the halo fixation device. J Bone Joint Surg Am 68:320-325, 1986

4. Goodman ML, Nelson PB: Brain abscess complicating the use of a halo orthosis. Neurosurgery 20:27-30, 1987

5. Humbyrd DE, Latimer FR, Lonstein JE, Samberg LC: Brain abscess as a complication of halo traction. Spine 6:365-368, 1981

6. Kameyama O, Ogawa K, Suga T, Nakamura T: Asymptomatic brain abscess as a complication of halo orthosis: report of a case and review of the literature. J Orthop Sci 4:39-41, 1999

7. Nottmeier EW, Bondurant CP: Delayed onset of generalized tonic-clonic seizures as a complication of halo orthosis. Case report. J Neurosurg 92 (2 Suppl):233-235, 2000

8. Olson RS: Halo skeletal traction pin site care: toward developing a standard of care. Rehabil Nurs 21:243-246, 257, 1996

9. Papagelopoulos PJ, Sapkas GS, Kateros KT, Papadakis SA, Vlamis JA, Falagas ME: Halo pin intracranial penetration and epidural abscess in a patient with a previous cranioplasty: case report and review of the literature. Spine 26:E463-E467, 2001

10. Perry J, Nickel VL: Total cervical spine fusion for neck paralysis. J Bone Joint Surg Am 41:37-60, 1959

11. Quiñones-Hinojosa A, Chi JH, Manley GT: Emergent placement of halo orthosis after a traumatic cervical injury leading to a cerebral abscess. J Trauma 62: E11-E13, 2007

12. Ray A, Iyer RV, King AT: Cerebral abscess as a delayed complication of halo fixation. Acta Neurochir (Wien) 148:1015-1016, 2006

13. Rizzolo SJ, Piazza MR, Cotler JM, Hume EL, Cautilli G, O’Neill DK: The effect of torque pressure on halo pin complication rates. A randomized prospective study. Spine 18:2163-2166, 1993

14. Rosenblum D, Ehrlich V: Brain abscess and psychosis as a complication of a halo orthosis. Arch Phys Med Rehabil 76: 865-867, 1995

15. Saeed MU, Dacuycuy MA, Kennedy DJ: Halo pin insertion-associated brain abscess: case report and review of literature. Spine 32:E271-E274, 2007

16. Victor DI, Bresnan MJ, Keller RB: Brain abscess complicating the use of halo traction. J Bone Joint Surg Am 55:635-639, 1973

17. Williams FH, Nelms DK, McGaharan KM: Brain abscess: a rare complication of halo usage. Arch Phys Med Rehabil 73:490-492, 1992

Manuscript submitted February 15, 2008.

Accepted March 31, 2008.

Address correspondence to: Meic H. Schmidt, M.D., Department of Neurosurgery, University of Utah, 175 N. Medical Drive East, Salt Lake City, Utah 84132. email: meic.schmidt@hsc.utah.edu. 